\title{
ASSOCIATION BETWEEN DEMOGRAPHIC CHARACTERISTICS AND COMPLIANCE OF STROKE PATIENTS TO PRESCRIBED UNSUPERVISED HOME EXERCISE PROGRAMMES
}

\author{
Journal website at; \\ http://mrtbjournal.org/index.php/njmr/issue/current/showToc \\ 'ADENIYI AF, 'ZANDAM HU \\ 'Department of Physiotherapy, Faculty of Clinical Sciences, \\ College of Medicine, University of Ibadan, Ibadan, Nigeria. \\ ${ }^{2}$ Department of Physiotherapy, Faculty of Medicine, \\ Bayero University, Kano, Nigeria. \\ Correspondence to: \\ A. F.Adeniyi \\ E-mail: adeniyifatai@yahoo.co.uk, fadeniyi@comui.edu.ng \\ Tel: $+234-8057325888$
}

\begin{abstract}
Background: Recovery of stroke patients to the pre-stroke status is not usually spontaneous and rehabilitation efforts continue beyond the sub-acute phase usually after hospital discharge.
\end{abstract}

Objective: To investigate whether stroke patients recuperating at home complied with prescribed Unsupervised Home Exercise Programme (UHEP) and what demographic characteristics were associated with noncompliance.

Methods: We administered questionnaires assessing full compliance and demographic characteristics of 108 consenting stroke patients attending the Outpatient Physiotherapy Clinics of two hospitals in Kano, Nigeria.

Results: Seventy-four percent of the participants were non-compliant with their UHEP. Stroke over one year with Odds Ratio $(\mathrm{OR})=2.1,95 \% \mathrm{CI}=1.823-3.315$, polygamy ( $\mathrm{OR}=3.1,95 \% \mathrm{CI}=2.661-5.012)$, occupying rented apartment $(\mathrm{OR}=10.8,95 \% \mathrm{CI}=2.037-28.319)$ and living with non-family members $(\mathrm{OR}=4.6,95 \% \mathrm{CI}=1.324-11.765)$ were significant predictors of non-compliance while gender, age group, marital status, education, monthly income and building type were not significant predictors ( $P>0.05$ ).

Conclusions: Most patients did not comply with their UHEP. Some of the socio-demographic factors responsible were duration of the stroke above one year, polygamy, living in rented apartment and with non-family members. Risk factors for non-compliance should be adequately considered when planning UHEP for stroke patients in order to enhance compliance.

Keywords: Self exercise, Hemiplegia, Physical therapy

\section{INTRODUCTION}

Rehabilitation after stroke has been described as a process in which the patient and the healthcare system, through interaction and negotiation, try to reach agreement about activities to be emphasised and goals to be pursued (Bendz, 2000). A review of studies comparing rehabilitation programmes administered in different environments concluded that therapy-based rehabilitation services for patients with stroke living at home improved their ability to perform unsupervised personal activities of daily living and reduced the risk of deterioration of their abilities (Ward et al., 2003). Patients undergoing rehabilitation at home took the initiative and expressed their goals more often than those undergoing hospital rehabilitation, thus, unsupervised rehabilitation at home seemed to empower patients (Holmqvist and von Koch, 2001). Advocates of home-based stroke rehabilitation suggest several advantages: satisfying patient choice, reducing the risks associated with inpatient care through reductions in length of hospital stay, the home setting being more focused toward rehabilitation outcomes, and savings in direct costs (Lafferty, 1996).

Rehabilitation services for stroke survivors are 
increasingly constrained by cost concerns, with pressure to discharge individuals from acute rehabilitation earlier when recovery and function have not yet stabilized (Duncan et al., 2003). Accordingly, effective and costefficient therapeutic interventions are needed to help promote cardiovascular fitness, improve endurance, and minimize chronic neuromuscular impairments due to disease or disuse in stroke survivors beyond the sub-acute post-stroke rehabilitation period (Smith et al., 1999). Stroke patients usually make private arrangement with experts for these post-stroke rehabilitations. However, not many stroke patients in the developing countries can afford rehabilitation services under the supervision of rehabilitation experts including physiotherapists, speech and occupational therapists. In view of this, such patients usually rely on the few outpatient appointments offered them at public hospitals, which is usually inadequate to meet their health needs. In order to make up for this shortfall, the physiotherapists attending to these patients usually prescribe a range of Unsupervised Home Exercise Programmes (UHEP) to be carried out by the patients on their own at home bearing in mind their health capacity to do so at that period in time. This study was therefore designed to investigate whether these patients complied or not with the UHEP and what socio-demographic characteristics were associated with their non-compliance.

\section{MATERIALSANDMETHODS}

This research was a cross-sectional survey of stroke patients undergoing rehabilitation programmes at the Physiotherapy Clinics of Aminu Kano Teaching Hospital (AKTH) Kano, and Murtala Mohammed Specialist Hospital, Kano, Nigeria. All stroke patients who were undergoing rehabilitation on out-patient basis at the Physiotherapy Clinics were assessed for eligibility by the authors, including being a patient undergoing out-patient physiotherapy treatment at the study centres, not visiting other physiotherapy clinics for treatment, receiving home exercise prescriptions and willingness to give informed consent. All the stroke patients who met the eligibility criteria during the study period were consecutively enrolled from the Physiotherapy Clinics.

The selected demographic characteristics studied included age, gender, marital status, type of marriage (monogamy/polygamy), living with family or non-family and duration of stroke. Others were educational attainment, employment status, monthly income, type of building (bungalow/storey), residential ownership, settlement (urban/rural) and opinion of the patient about the UHEP. These questions were originally verified by practicing Physiotherapists who were involved in the management of stroke patients. Prior to the study, ethical approval was obtained from the Ethical Committee on Research of AKTH, Kano.

Data Collection: The participants were asked to fill the questionnaires and encouraged to supply answers to each of the questions. Assistance was provided where necessary. The questions asked were on gender, age, duration of stroke which was from the day of onset to the time of this study recorded in months. We also asked questions on the marital status of the participant (married, divorced, separated and widowed) and also sought to know whether marriage was monogamous or polygamous as at study time. There were questions also on the kind of settlement where the participant was residing with options of urban or rural settlement; whether the participant was living in a rented or owned apartment, bungalow or a storey building and whether living with family or non-family members. We also probed into whether the participant was educated or not, employment status (employed or unemployed) and monthly income of the participant. Finally, we sought to know the opinion of the participant about the UHEP; (whether it was necessary, waste of time or viewed as "I don't have a choice") and if the participant had candidly complied or not with the prescribed UHEP on the basis of frequency, duration and intensity.

The demographic characteristics of the participants were summarized using frequencies and percentages. A forward stepwise variable selection of the binary logistic regression model was used to indicate the demographic characteristics that significantly contributed to the model. Odds ratios for risk of non-compliance were further determined. The analyses were performed with SPSS 15.0 for Windows (SPSS Inc., Chicago).

\section{RESULTS}

The summary of the demographic characteristics of the participants are as shown in Table 1. With mean age of $58 \pm 6.21$ years, most of the participants $(37.0 \%)$ were between the ages of 60-69 years with the least occurring in the age groups $30-39$ years $(1.9 \%)$ and $=90$ years $(0.9 \%)$. Of the 108 participants, 83 were males, $63.9 \%$ married polygamists and most of the participants $(9.3 \%)$ had had the stroke for about 9 months. Most of the participants (74.1\%) reported not complying with the instructions given

Table 1: Frequency distribution of socio-demographic characteristics of the participants

\begin{tabular}{llcc}
\hline Variable & $\begin{array}{l}\text { Modal } \\
\text { group }\end{array}$ & Frequency & Percentage \\
\hline Sex & Male & 83 & 76.9 \\
Age group(Years) & $60-69$ & 40 & 37.0 \\
Duration of stroke & 9 months & 10 & 9.3 \\
Settlement type & Urban & 81 & 75.0 \\
Residence Ownership & Rented & 78 & 73.1 \\
Building Type & Bungalow & 65 & 60.2 \\
Marital status & Married & 72 & 66.7 \\
Marriage Type & Polygamy & 41 & 37.9 \\
Cohabitation & Spouse + & & \\
& relatives & 35 & 32.4 \\
Education & Nil & 40 & 37.0 \\
Monthly income (Naira) & $30-59,000$ & 34 & 31.5 \\
Compliance & No & 80 & 74.1 \\
Opinion & Necessary & 64 & 59.1 \\
\hline
\end{tabular}


Table 2: Parameter estimate table summarizing the effects of each predictor in the model

\begin{tabular}{llllllll}
\hline Variable & B & SE & Wald & df & P-value & Odds Ratio & 95\% CI \\
\hline Duration of stroke (> 1 year) & 3.512 & 0.033 & 27.215 & 1 & 0.001 & 2.134 & $1.823-3.315$ \\
Marriage type (polygamy) & 0.681 & 1.071 & 18.141 & 1 & 0.041 & 3.148 & $2.661-5.012$ \\
Ownership of residence (rented) & 2.380 & 0.851 & 7.817 & 1 & 0.005 & 10.807 & $2.037-28.319$ \\
Location of residence (rural) & -2.471 & 3.525 & 9.716 & 1 & 0.001 & 0.431 & $0.163-0.772$ \\
Cohabitation (with non-family members) & 2.992 & 1.240 & 3.061 & 1 & 0.007 & 4.655 & $1.324-11.765$ \\
Employment status (employed) & -1.081 & 0.069 & 17.869 & 1 & 0.035 & 0.563 & $0.364-0.816$ \\
Opinion (necessary) & -3.621 & 1.232 & 8.644 & 1 & 0.003 & 0.727 & $0.498-0.886$ \\
\hline
\end{tabular}

$\mathrm{B}=$ Standardised coefficient; $\mathrm{SE}=$ Standard Error, Wald = Wald Statistics; $\mathrm{df}=$ degrees of freedom; CI = Confidence Interval

by their physiotherapists on the basis of frequency, duration and intensity of UHEP. However, $59.1 \%$ of them agreed that UHEP were necessary for their continued improvement.

The forward stepwise regression statistics of the demographic characteristics shows that duration of stroke, settlement type, marriage type (monogamy or polygamy), cohabitation (living with others), residence ownership, employment status and opinion of patient about UHEP contributed to the model $(\mathrm{P}<0.05)$ while gender, age group, marital status, education, income and building type were not significantly useful to the model $(P>0.05)$ and were subsequently eliminated. The parameter estimate (Table 2) presents the useful predictors and coefficients used to make predictions. Non-compliance was significantly associated with duration of stroke longer than one year $(\mathrm{OR}=2.1,95 \% \mathrm{CI}=1.823-3.315)$, polygamy $(\mathrm{OR}=3.1,95 \%$ $\mathrm{Cl}=2.661-5.012)$, living in rented apartment $(\mathrm{OR}=10.8,95 \%$ $\mathrm{CI}=2.037-28.319)$ and living with non-family members $(\mathrm{OR}=4.6,95 \% \mathrm{CI}=1.324-11.765)$. Non-compliance was less likely if patient was living in rural community $(\mathrm{OR}=0.4$, 95\% CI $=0.163-0.772)$, was employed $(\mathrm{OR}=0.6,95 \%$ $\mathrm{CI}=0.364-0.816$ ) and viewed the UHEP as important for recovery $(\mathrm{OR}=0.03,95 \% \mathrm{Cl}=0.498-0.886)$.

\section{DISCUSSION}

This study found a high level of non-compliance to prescribed Unsupervised Home Exercise Programme (UHEP) by stroke patients undergoing rehabilitation at the research centres. Eighty percent of the participants confessed non-compliance with the specific UHEP prescribed for them by their physiotherapists. The significant risk factors for non-compliance in these patients were duration of stroke longer than a year, being married into polygamy, residing in rented apartment and living with non-family members. We further found that noncompliance was significantly less likely to occur in our stroke patients who were residing in the rural areas (or less city), who were employed and who viewed unsupervised home exercise programmes as crucial to their recovery. However, gender of the participants, their age groups, marital status, whether educated or not, monthly income and whether residing in a bungalow or storey building did not significantly predict non-compliance.

Home-based rehabilitation after stroke is essential because it has been shown to considerably shorten the length of stay in hospital by nearly 1 to 2 weeks on average, without compromising patient safety or functional outcomes for survivors of stroke (Anderson et al., 2000). Recently in a study carried out elsewhere, it was found that both supervised exercise programs and unsupervised programs after initial supervised instruction were both associated with physical benefits including balance and mobility functions that were retained for 1 year, although supervised programs showed trends to greater improvements in self-reported gains (Olney et al., 2006). However, in spite of the benefits and effectiveness of UHEP for stroke patients, our study showed that most of them were not compliant with the programmes.

The increased risk of non-compliance to UHEP attributable to stroke duration of more than a year in our study could be due to lack of knowledge about stroke recovery, frustration, and lost of interest in the UHEP especially when the patient felt "making appreciable improvement" had ceased. These attitudes were likely to set in as the duration of disability increased, further worsening the functional status of the patients. Functional consequences associated with long-term motor deficits have been documented to predispose majority of stroke survivors to a generally sedentary lifestyle with concomitant deconditioning, disuse atrophy, and sarcopenia, thereby contributing to further declines in activities of daily living and disability status (Smith et al., 1999).

The fact that each of living with non-family members and polygamous marriage settings were significant predictors of non-compliance to UHEP could best be described as lacking in the midst of plenty. These risk factors underscored the possibility of the stroke patients living with people who are unfortunately less interested in their welfare. These risk factors may bring about patient isolation and want of assistance because entities in the domestic social support organ of the patient lacked genuine commitment to assist the patient as a result of major or remote discord among the family members. Assistance with UHEP and other activities of daily living from individuals around the paticnt is very vital fur their recovery. Although nearly $14 \%$ of stroke survivors achieve full recovery in physical function, between $25 \%$ and $50 \%$ have been shown to require at least some assistance with 
activities of daily living while half experience severe longterm effects such as partial paralysis (Gordon et al., 2004). Hence, lack of home support and social isolation may contribute to poorer post-stroke outcomes due to poor compliance to therapy regimen, depression, and stress (Boden-Albala et al., 2005). Therefore in order to facilitate optimal outcomes from an exercise-based stroke rehabilitation program, an assessment of familial support should be undertaken since nearly $80 \%$ of all patients needing home care after an illness receive at least part of their care from a family member (Gordon et al., 2004).

In this study, non-compliance to UHEP was significantly less likely in patients residing in the rural than urban locations. We postulate this might be because stroke patients living in the rural areas have adequate serene environment unlike the urban settlement where noncompliance may be because of perceived lack of privacy vis-à-vis many available potential distractions such as availability of multi-channels television views and social visitations. In a slight contrast, a study in the United Kingdom, though on normal elderly adults found that while overall levels of activity were similar for both urban and rural residents, the structure of activities differed, with the urban sample reporting significantly greater time spent walking (Morgan et al., 2000). Non-compliance was also found to be less likely in our study participants who were gainfully employed. Involuntary job loss for instance is a major life event associated with social, economic, behavioural, and health outcomes, for which older workers are at elevated risk (Gallo et al., 2006). Perceived fear of joblessness may have been responsible for better compliance to UHEP by the stroke survivors who were employed hence returning to their jobs could be the strong motivation.

The fact that some participants viewed UHEP as unimportant or a mere waste of time could mean that they were ill-informed or demotivated about the benefit of the programme. According to Holmqvist and von Koch (2001) there is consensus among professionals in stroke rehabilitation that the patient's degree of motivation will influence the outcome of an intervention. Consequently, an important element of any proposed intervention should be an assessment of what the patient is motivated to achieve as well as the promotion of a high level of motivation. Motivation may be influenced by both personal factors (such as age, gender, personality, educational and social background, experience, coping capacity, health status, and lifestyle) and environmental factors, which together shape the physical, social, and attitudinal context for rehabilitation (Maclean et al., 2000, Holmqvist and von Koch, 2001). In addition, a diminished self-efficacy, greater dependence on others for activities of daily living, and reduced ability for normal societal interactions can have a profound negative psychological impact on the person (Gordon et al., 2004).

This study is of major clinical importance because it has revealed how ordinary data we obtain from patients on routine clinical assessment can determine compliance to UHEP which is an important factor for recovery. This will help rehabilitation experts in typical developing countries identify patients who are not likely to comply and subsequent steps will be taken to address such risk factors. The results of this study demonstrate that stroke patients in a developing country need to be adequately educated on the need for conscientious participation in prescribed UHEP for the purpose of recovery and health promotion. This is especially necessary because stroke patients will have to be discharged home at a point during recovery and most of them will only do well if the subsequent medical management is augmented with UHEP. In creating and implementing any type of recovery program for the stroke survivor, it is necessary to be aware of cultural differences that may affect acceptance or adherence and the integration of patient and family cultural norms will also help create a program that is more likely to succeed over the long term (Gordon et al., 2004).

An important limitation in this study was that report on compliance was supplied solely by the participants as we did not have the opportunity to confirm the genuineness of their claims regarding the UHEP. We however emphasized the importance of responding in affirmative to the question seeking their compliance only if they carried out the UHEP on the basis of prescribed frequency, intensity and duration. The non-compliance to UHEP observed in these patients may not be due only to the included variables. Potential confounders such as post stroke depression, lack of motivation and psychosocial variables may exist. Strength of this study however is that it has provided the much needed information on compliance to UHEP by stroke patients especially of a sample from developing countries and economies. This is because observations from this kind of study may have socio-economic connotations and previous studies on this subject were mostly carried out from developed countries making extrapolation of findings difficult. It also shows how sociodemographic characteristics could influence their participation in a rehabilitation activity that is supposed to make up for the periods when they are not in the hospital setting or under the supervision of a trained physical therapist.

In conclusion, we report that most of the stroke patients undergoing rehabilitation on outpatient basis were non-compliant with their prescribed Unsupervised Home Exercise Programme (UHEP) and the significant predictors of non-compliance were duration of stroke longer than one year, polygamy, not employed, living with non-family members, living in rented apartments, urban settlement and not viewing the UHEP as necessary for recovery. Before the development and prescription of UHEP, effective investigation and analyses of the patient's demographic characteristics is important to identify patients at risk for non-compliance and to analyze what form of strategy may be adopted to achieve compliance in these people. These strategies among others may include reinforcement of 
potential benefits of the UHEP and psychotherapy. Further research is required to confirm that indeed some demographic characteristics such as sex, age group, marital status, monthly income, educational attainment and living in a bungalow or storey building do not predict compliance to UHEP in stroke patients. Studies that will look into compliance to UHEP from the point of view of depression and motivation in this group of patients will also be relevant.

\section{REFERENCES}

- Anderson C, Rubenach S, Mhurchu CN, Clark M, Spencer C, Winsor A. (2000). Home or hospital for stroke rehabilitation? Results of a randomized controlled trial I: Health outcomes at 6 months. Stroke. 31(5): 1024-1031.

- Bendz M. (2000). Rules of relevance after stroke. Social Science and Medicine. 51(5): 713-723.

- Boden-Albala B, Litwak E, Elkind MSV, Rundek T, Sacco R.L. (2005). Social isolation and outcomes post stroke. Neurology. 64(11): 1888-1892.

- Duncan P, Studenski S, Richards L, Gollub S, Lai SM, Reker D, Perera S, Yates J, Koch V, Rigler S, Johnson D. (2003). Randomized clinical trial of therapeutic exercise in subacute stroke. Stroke. 34(9): 2173-2180.

- Gallo WT, Teng HM, Falba TA, Kasl SV, Kumholz HM, Bradley BH (2006). The impact of late career job loss on myocardial infàrction and stroke: a 10 year follow up using the health and retirement survey. Occupational and Environmental Medicine. 63(10): 683-687.

- Gordon NF, Gulanick M, Costa F, Fletcher G, Franklin BA, Roth EJ, Shephard T. (2004). Physical activity and exercise recommendations for stroke survivors: An American Heart Association Scientific Statement from the Council on Clinical
Cardiology, Subcommittee on Exercise, Cardiac Rehabilitation, and Prevention; the Council on Cardiovascular Nursing; the Council on Nutrition, Physical Activity, and Metabolism; and the Stroke Council. Stroke. 35(5): $1230-1240$.

- Holmqvist LW, von Koch L. (2001). Environmental factors in stroke rehabilitation Being in hospital itself demotivates patients. British Medical Journal. 322(7301): 1501-1502.

- Lafferty G. (1996). Community-based alternatives to hospital rehabilitation services: a review of the evidence and suggestions for approaching future evaluations. Reviews in Clinical Gerontology. 6(2): 183-194.

- Maclean N., Pound P., Wolfe C., Rudd A. (2000). Qualitative analysis of stroke patients' motivation for rehabilitation. British Medical Joumal. 321(7268): 1051-1054.

- Morgan K., Armstrong G, Huppert F., Brayne C., Solomou W. (2000). Health ageing in urban and rural Britain: a comparison of exercise and diet. Age and Ageing. 29(4): 341-348.

- Olney S.J., Nymark J., Brouwer B., Culham E., Day A., Heard J., Henderson M., Parvataneni K. (2006). A randomized controlled trial of supervised versus unsupervised exercise programs for ambulatory stroke survivors. Stroke. 37(2): 476-481.

- Smith G. V., Silver K.H.C., Goldberg A.P., Macko R.F. (1999). Task oriented exercise improves hamstring strength and spastic reflexes in chronic stroke patients. Stroke. 30(10): 2112-2118.

- Ward D., Severs M., Dean T., Brooks N. (2003). Care home versus hospital and own home environments for rehabilitation of older people (Cochrane Review). Cochrane Database (2):CD003164. 